\title{
Moduli spaces of orthogonal and symplectic bundles over an algebraic curve
}

\author{
Olivier Serman
}

\begin{abstract}
We prove that, given a smooth projective curve $C$ of genus $g \geqslant 2$, the forgetful morphism $\mathcal{M}_{\mathbf{O}_{r}} \longrightarrow \mathcal{M}_{\mathbf{G L}_{r}}$ (respectively $\mathcal{M}_{\mathbf{S p}_{2 r}} \longrightarrow \mathcal{M}_{\mathbf{G L}_{2 r}}$ ) from the moduli space of orthogonal (respectively symplectic) bundles to the moduli space of all vector bundles over $C$ is an embedding. Our proof relies on an explicit description of a set of generators for the polynomial invariants on the representation space of a quiver under the action of a product of classical groups.
\end{abstract}

\section{Introduction}

Let $C$ be a smooth, irreducible, projective algebraic curve of genus $g \geqslant 2$ over an algebraically closed field $k$ of characteristic 0 . If $G$ is a reductive group over $k$, we denote by $\mathcal{M}_{G}$ the moduli space of semi-stable principal $G$-bundles on $C$.

We focus here on the case $G=\mathbf{S O}_{r}$, which amounts to considering the moduli space of semistable orthogonal bundles of rank $r$ with an orientation. It is a normal projective variety, composed of two connected components distinguished by the second Stiefel-Whitney class. This space is related to the moduli space $\mathcal{M}_{\mathbf{S L}_{r}}$ of vector bundles of rank $r$ and trivial determinant on $C$ through the forgetful morphism $\mathcal{M}_{\mathbf{S O}_{r}} \longrightarrow \mathcal{M}_{\mathbf{S L}_{r}}$ which sends any $\mathbf{S O}_{r}$-bundle to its underlying vector bundle. It is natural to ask whether this map is a closed embedding. In fact, when $r$ is even, it even fails to be injective, and it is therefore more convenient to ask the same question about $\mathcal{M}_{\mathbf{O}_{r}} \longrightarrow \mathcal{M}_{\mathbf{G L}_{r}}$.

In the same way we consider the forgetful morphism $\mathcal{M}_{\mathbf{S p}_{2 r}} \longrightarrow \mathcal{M}_{\mathbf{S L}_{2 r}}$ defined on the moduli space of symplectic bundles of rank $2 r$ on $C$.

Our main theorem may be stated as follows.

Theorem (Main theorem).

(i) The forgetful morphism $\mathcal{M}_{\mathbf{O}_{r}} \longrightarrow \mathcal{M}_{\mathbf{G L}_{r}}$ is an embedding.

(ii) When $r$ is odd, $\mathcal{M}_{\mathbf{S O}_{r}} \longrightarrow \mathcal{M}_{\mathbf{S L}_{r}}$ is again an embedding; while, when $r$ is even, it is a 2-sheeted cover onto its image.

(iii) The forgetful morphism $\mathcal{M}_{\mathbf{S p}_{2 r}} \longrightarrow \mathcal{M}_{\mathbf{S L}_{2 r}}$ is also an embedding.

We give the full proof for the orthogonal case, and sketch the obvious modifications required by the symplectic one.

We consider in the first section the injectivity of $\mathcal{M}_{\mathbf{S O}_{r}} \longrightarrow \mathcal{M}_{\mathbf{S L}_{r}}$ : this comes down to an easy comparison of the equivalence relations between $\mathbf{S O}_{r}$-bundles and vector bundles which define the closed points of the corresponding moduli spaces. We then check that the tangent maps of $\mathcal{M}_{\mathbf{O}_{r}} \longrightarrow \mathcal{M}_{\mathbf{G L}_{r}}$ are injective. This differential point of view is much more involved: it relies on

Received 27 September 2006, accepted in final form 16 July 2007, published online 14 March 2008. 2000 Mathematics Subject Classification 14H60 (primary), 14F05, 14L30, 16G20 (secondary).

Keywords: moduli spaces, orthogonal bundles, symplectic bundles, representations of quivers.

This journal is (C) Foundation Compositio Mathematica 2008. 


\section{O. Serman}

Luna's étale slice theorem, which naturally leads to the consideration of representations of quivers. To carry our discussion to its end we need an auxiliary result relative to the invariant theory of these representations for the action of a product of classical groups: this is the aim of the second section (note that a characteristic-free proof of this result can be found in [Lop06]). In the third section we show first how this computation results in our main theorem. We then give a few complements about the local structure of the moduli space $\mathcal{M}_{\mathbf{O}_{r}}$, in the same way as Laszlo did with $\mathcal{M}_{\mathbf{G L}_{r}}$ (see [Las96]).

\section{On the injectivity of $\mathcal{M}_{\mathrm{SO}_{r}} \longrightarrow \mathcal{M}_{\mathrm{SL}_{r}}$}

In this section we study the injectivity of the forgetful map $\mathcal{M}_{\mathbf{S O}_{r}} \longrightarrow \mathcal{M}_{\mathbf{S L}_{r}}$, which is already known to be finite (e.g. by [BS02, Theorem 8.5]).

1.1 The closed points of $\mathcal{M}_{G}$ are in a one-to-one correspondence with the set of equivalence classes of semi-stable $G$-bundles (cf. [Ram96]). When $G=\mathbf{S L}_{r}$, one easily recovers from this notion Seshadri's definition of $S$-equivalence for vector bundles. In the orthogonal case we just need to recall the following two facts (see [Ram96, Corollary 3.15.1]): any closed point corresponds to a unitary $\mathbf{S O}_{r}$-bundle $P$, well defined up to isomorphism; and the underlying vector bundle $P\left(\mathbf{S L}_{r}\right)=P \times{ }^{\mathbf{S O}_{r}} \mathbf{S L}_{r}$ is a unitary vector bundle, i.e. a polystable vector bundle.

1.2 Two unitary $\mathbf{S O}_{r}$-bundles $P$ and $P^{\prime}$ are sent to the same point of $\mathcal{M}_{\mathbf{S L}_{r}}$ if and only if they are both obtained from reduction of structure group to $\mathbf{S O}_{r}$ of the same polystable vector bundle $E$. Such a reduction amounts to a section of $E / \mathbf{S O}_{r} \rightarrow C$, and two of them give isomorphic $\mathbf{S O}_{r^{-}}$ bundles if and only if they are conjugated by the action of $\operatorname{Aut}_{\mathbf{S L}_{r}}(E)$ on $\Gamma\left(C, E / \mathbf{S O}_{r}\right)$. Elements of $\Gamma\left(C, E / \mathbf{S O}_{r}\right)$ correspond to isomorphisms $\iota: E \stackrel{\sim}{\longrightarrow} E^{*}$ such that $\iota^{*}=\iota$ and $\operatorname{det} \iota$ is the square of the trivialization of det $E$ inherited from the $\mathbf{S L}_{r}$-torsor structure. The action of $\operatorname{Aut}_{\mathbf{S L}}(E)$ is simply

$$
(f, \iota) \in \operatorname{Aut}_{\mathbf{S L}_{r}}(E) \times \Gamma\left(C, E / \mathbf{S O}_{r}\right) \mapsto f^{*} \iota f .
$$

Since $E$ is polystable, $\operatorname{Aut}_{\mathbf{G L}_{r}}(E)$ acts transitively on the set $\Gamma\left(C, E / \mathbf{O}_{r}\right)$ of all symmetric isomorphisms from $E$ onto $E^{*}$ : indeed, the Jordan-Hölder filtration allows us to split $E$ as

$$
E=\bigoplus_{i=1}^{n_{1}}\left(F_{i}^{(1)} \otimes V_{i}^{(1)}\right) \oplus \bigoplus_{j=1}^{n_{2}}\left(F_{j}^{(2)} \otimes V_{j}^{(2)}\right) \oplus \bigoplus_{k=1}^{n_{3}}\left(\left(F_{k}^{(3)} \oplus F_{k}^{(3) *}\right) \otimes V_{k}^{(3)}\right),
$$

for some finite-dimensional vector spaces $V_{i}^{(l)}$ and some mutually non-isomorphic orthogonal (respectively symplectic, respectively non-isomorphic to their dual) stable vector bundles, $F_{i}^{(1)}$ (respectively $F_{j}^{(2)}$, respectively $F_{k}^{(3)}$ ) on $C$. A symmetric isomorphism $E \rightarrow E^{*}$ is thus equivalent to the data of some orthogonal (respectively symplectic, respectively non-degenerate) forms on each $V_{i}^{(1)}$ (respectively $V_{j}^{(2)}$, respectively $V_{k}^{(3)}$ ). The action of

$$
\operatorname{Aut}_{\mathbf{G L}_{r}}(E)=\prod_{i=1}^{n_{1}} \mathbf{G} \mathbf{L}\left(V_{i}^{(1)}\right) \times \prod_{j=1}^{n_{2}} \mathbf{G L}\left(V_{j}^{(2)}\right) \times \prod_{k=1}^{n_{3}}\left(\mathbf{G L}\left(V_{k}^{(3)}\right) \times \mathbf{G} \mathbf{L}\left(V_{k}^{(3)}\right)\right)
$$

on the set of these collections is obviously transitive.

Remark 1.3. (i) The preceding discussion gives a precise description of unitary $\mathbf{O}_{r}$-bundles, which will be used later: these are orthogonal bundles $P$ whose underlying vector bundle $E$ can be decomposed as in (1.2.1), $\left(V_{i}^{(1)}\right)_{i}$ being some quadratic spaces, $\left(V_{j}^{(2)}\right)_{j}$ some symplectic spaces, and $\left(V_{k}^{(3)}\right)_{k}$ some vector spaces carrying a non-degenerate bilinear form (note that the bundles $F_{k}^{(3)} \oplus F_{k}^{(3)} *$ have been tacitly endowed with the hyperbolic form). 


\section{ORTHOGONAL AND SYMPLECTIC BUNDLES ON CURVES}

The subgroup $\operatorname{Aut}_{\mathbf{O}_{r}}(P) \subset \operatorname{Aut}_{\mathbf{G L}_{r}}(E)$ of all orthogonal isomorphisms of the bundle $P$ is then easily described: it is isomorphic to

$$
\prod_{i=1}^{n_{1}} \mathbf{O}\left(V_{i}^{(1)}\right) \times \prod_{j=1}^{n_{2}} \mathbf{S} \mathbf{p}\left(V_{j}^{(2)}\right) \times \prod_{k=1}^{n_{3}} \mathbf{G} \mathbf{L}\left(V_{k}^{(3)}\right),
$$

where $\mathbf{G} \mathbf{L}\left(V_{k}^{(3)}\right)$ is identified with its image in $\mathbf{G L}\left(V_{k}^{(3)}\right) \times \mathbf{G} \mathbf{L}\left(V_{k}^{(3)}\right)$ by the morphism $g \mapsto\left(g,{ }^{\mathrm{t}} g^{-1}\right)$.

(ii) Coming back to Ramanathan's algebraic characterization of the representative of the equivalence class of a semi-stable $\mathbf{S O}_{r}$-bundle $P$ (which is obtained from a suitable reduction of the structure group of $P$ to a parabolic subgroup of $\mathbf{S O}_{r}$, see [Ram96, Proposition 3.12]), we note that the unitary bundle corresponding to the class of $P$ is defined as the oriented orthogonal graded object $\operatorname{gr} P$ associated to any filtration of the underlying vector bundle $E=P\left(\mathbf{S L}_{r}\right)$,

$$
0=E_{0} \subset E_{1} \subset \cdots \subset E_{l} \subset E_{l}^{\perp} \subset \cdots \subset E_{1}^{\perp} \subset E,
$$

where the $E_{i}$ are isotropic subbundles such that $E_{i} / E_{i-1}$ is a stable vector bundle (of degree 0 ) and $E_{l}^{\perp} / E_{l}$ a stable (oriented) orthogonal bundle. By [Ram81, Proposition 4.5] the latter splits as a direct orthogonal sum of mutually non-isomorphic stable bundles: this exactly means that $(\operatorname{gr} P)\left(\mathbf{S L}_{r}\right)$ is the unique polystable bundle $S$-equivalent to $E$, which gives another proof of the fact that, if $P$ is a unitary $\mathbf{S O}_{r}$-bundle, the vector bundle $P\left(\mathbf{S L}_{r}\right)$ is polystable.

Note that this description of closed points of $\mathcal{M}_{\mathbf{S O}_{r}}$ has already been given, for example in [Bho84].

1.4 We deduce from Paragraph 1.2 that any two elements of $\Gamma\left(C, E / \mathbf{S O}_{r}\right)$ must be conjugate under the action of $\operatorname{Aut}_{\mathbf{G} \mathbf{L}_{r}}(E)$, by an automorphism whose determinant equals \pm 1 . When $r$ is odd, $-\mathrm{id}_{E}$ is an $\mathbf{O}_{r}$-isomorphism which exchanges the orientation, and the action of $\operatorname{Aut}_{\mathbf{S L}_{r}}(E)$ on $\Gamma\left(C, E / \mathbf{S O}_{r}\right)$ is still transitive. On the contrary, when $r$ is even, this action fails to remain transitive. For example let $F$ be a vector bundle of rank $r / 2$, non-isomorphic to its dual, and consider the two orthogonal bundles $F \oplus F^{*}$ and $F^{*} \oplus F$, equipped with the standard hyperbolic pairing: these bundles cannot be $\mathbf{S O}_{r}$-isomorphic (in fact, any orthogonal automorphism of $F \oplus F^{*}$ must preserve the orientation). We have proven so far the following proposition.

Proposition 1.5. When $r$ is odd, the map $\mathcal{M}_{\mathbf{S O}_{r}}(k) \longrightarrow \mathcal{M}_{\mathbf{S L}_{r}}(k)$ is injective; when $r$ is even, this is a finite map of degree 2 .

Remark 1.6. The distinction between the odd and even cases relies on the fact that the semi-direct product $1 \rightarrow \mathbf{S O}_{r} \rightarrow \mathbf{O}_{r} \rightarrow \mathbb{Z} / 2 \mathbb{Z} \rightarrow 0$ may be direct or not. Indeed, [Gir71, Proposition III 3.3.3] gives a general way to compute the fibres of the map (of pointed sets) $H_{\text {ét }}^{1}\left(C, \mathbf{S O}_{r}\right) \longrightarrow$ $H_{\text {ét }}^{1}\left(C, \mathbf{O}_{r}\right)$ (which forgets the orientation). These are exactly the orbits under a natural action of $H^{0}(C, \mathbb{Z} / 2 \mathbb{Z})=\mathbb{Z} / 2 \mathbb{Z}$ on $H_{\text {ét }}^{1}\left(C, \mathbf{S O}_{r}\right)$, for which stabilizers are easy to describe: the stabilizer of an $\mathbf{S O}_{r}$-bundle $P$ is the image of the map $\operatorname{Aut}_{\mathbf{O}_{r}}(P) \rightarrow \mathbb{Z} / 2 \mathbb{Z}$ obtained by twisting the quotient morphism $\mathbf{O}_{r} \rightarrow \mathbb{Z} / 2 \mathbb{Z}$ by $P$ (with respect to the action of $\mathbf{S O}_{r}$ by inner automorphisms) and taking global sections.

When $r$ is odd, the product $1 \rightarrow \mathbf{S O}_{r} \rightarrow \mathbf{O}_{r} \rightarrow \mathbb{Z} / 2 \mathbb{Z} \rightarrow 0$ is direct, and the map $H_{\text {ét }}^{1}\left(C, \mathbf{S O}_{r}\right) \longrightarrow$ $H_{\text {ét }}^{1}\left(C, \mathbf{O}_{r}\right)$ is automatically injective. But, as soon as $r$ is even, its section is no longer compatible with the action of $\mathbf{S O}_{r}$ by inner automorphisms; we have just chosen a bundle $E=F \oplus F^{*}$ such that $\operatorname{Aut}_{\mathbf{O}_{r}}(E)=\{\mathrm{id}\}$, whence the lack of injectivity.

In view of Remark 1.3 (i) we can give a complete description of $\mathcal{M}_{\mathbf{S O}_{r}}(k) \longrightarrow \mathcal{M}_{\mathbf{O}_{r}}(k)$ : a unitary orthogonal bundle $P$ with trivial determinant has two antecedents in $\mathcal{M}_{\mathbf{S O}}$ if and only if every orthogonal bundle $F_{i}^{(1)}$ appearing in the splitting (1.2.1) of its underlying vector bundle $P\left(\mathbf{G L}_{r}\right)$ has even rank. 


\section{O. SERMAN}

\section{Invariant theory of representations of quivers}

We refer the reader to [LBP90] for the notion of representations of a quiver $Q$ of given dimension $\alpha \in \mathbb{N}^{n}$. Let $Q$ stand for a quiver consisting of $n=n_{1}+n_{2}+2 n_{3}$ vertices

$$
s_{1}, \ldots, s_{n_{1}}, t_{1}, \ldots, t_{n_{2}}, u_{1}, u_{1}^{*}, \ldots, u_{n_{3}}, u_{n_{3}}^{*},
$$

and $\alpha \in \mathbb{N}^{n} \simeq \mathbb{N}^{n_{1}} \times \mathbb{N}^{n_{2}} \times(\mathbb{N} \times \mathbb{N})^{n_{3}}$ be an admissible dimension vector (that is, a vector such that $\alpha_{t_{j}}$ is even and $\left.\alpha_{u_{k}}=\alpha_{u_{k}^{*}}\right)$. We define $\Gamma_{\alpha}$ to be the group

$$
\Gamma_{\alpha}=\prod_{i=1}^{n_{1}} \mathbf{O}_{\alpha_{s_{i}}} \times \prod_{j=1}^{n_{2}} \mathbf{S p}_{\alpha_{t_{j}}} \times \prod_{k=1}^{n_{3}} \mathbf{G} \mathbf{L}_{\alpha_{u_{k}}}
$$

which is actually thought of here as a subgroup of $\mathbf{G L}(\alpha)=\prod_{i=1}^{n} \mathbf{G L}_{\alpha_{i}}$ via the inclusions $P \in$ $\mathbf{G L}_{\alpha_{u_{k}}} \mapsto\left(P,{ }^{\mathrm{t}} P^{-1}\right) \in \mathbf{G L}_{\alpha_{u_{k}}} \times \mathbf{G L}_{\alpha_{u_{k}^{*}}}$ for $k=1, \ldots, n_{3}$. The natural action of $\mathbf{G L}(\alpha)$ on the space $R(Q, \alpha)$ of all representations of $Q$ of dimension $\alpha$ restricts to an action of $\Gamma_{\alpha}$ on $R(Q, \alpha)$.

Le Bruyn and Procesi have shown in [LBP90] that the algebra $k[R(Q, \alpha)]^{\mathbf{G L}(\alpha)}$ of polynomial invariants is generated by traces along oriented cycles in the quiver $Q$. Following their proof, we produce here a set of generators for the algebra of invariants under the action of $\Gamma_{\alpha}$. The local study of the map $\mathcal{M}_{\mathbf{O}_{r}} \longrightarrow \mathcal{M}_{\mathbf{G} \mathbf{L}_{r}}$ made later rests heavily on this description.

\subsection{First fundamental theorem for $\mathrm{O}_{N} \times \mathrm{Sp}_{N^{\prime}}$}

In this section, we first adapt the argument of [ABP73, Appendix 1] to prove the first fundamental theorem of invariant theory for the group $\mathbf{O}_{N} \times \mathbf{S p}_{N^{\prime}}$, and then show, after [Pro76], how to infer from it a set of generators for the algebra of $\mathbf{O}_{N} \times \mathbf{S} \mathbf{p}_{N^{\prime}}$-invariants of $m$ matrices.

2.1.1 We will denote by $\mathrm{M}_{n}$ the space of $n \times n$ matrices. Let $M$ be the matrix

$$
M=\left(\begin{array}{cc}
I_{N} & 0 \\
0 & J_{N^{\prime}}
\end{array}\right)
$$

with

$$
J=\left(\begin{array}{cc}
0 & -I \\
I & 0
\end{array}\right)
$$

it represents a bilinear pairing, given as the standard orthogonal sum of a quadratic form of rank $N$ and a symplectic form of rank $N^{\prime}$. The key lemma of [ABP73] becomes (note that $\mathrm{M}_{N} \times \mathrm{M}_{N^{\prime}}$ is identified with its image in $\mathrm{M}_{N+N^{\prime}}$ ) the following.

Lemma 2.1.2. Any polynomial function $f:\left(\mathrm{M}_{N} \times \mathrm{M}_{N^{\prime}}\right)(k) \rightarrow k$ such that $f(B A)=f(A)$ for all $B \in \mathbf{O}_{N} \times \mathbf{S p}_{N^{\prime}}$ may be written $f: A \mapsto F\left({ }^{\mathrm{t}} A M A\right)$, with $F$ a polynomial map on $\left(\mathrm{M}_{N} \times \mathrm{M}_{N^{\prime}}\right)(k)$.

In other words, $f$ factors through the application

$$
\pi: A \in\left(\mathrm{M}_{N} \times \mathrm{M}_{N^{\prime}}\right)(k) \mapsto{ }^{\mathrm{t}} A M A \in\left(\mathrm{M}_{N} \times \mathrm{M}_{N^{\prime}}\right)(k) .
$$

Let $\Psi_{N, N^{\prime}}$ be its image, which is nothing other than the product of the space of symmetric $N \times N$ matrices and the space of antisymmetric $N^{\prime} \times N^{\prime}$ matrices. The restriction of $\pi$ to $\mathbf{G} \mathbf{L}_{N} \times \mathbf{G L}_{N^{\prime}}$ identifies the open subset $\Psi_{N, N^{\prime}}^{\circ}$ consisting of non-degenerate forms with the geometric quotient $\left(\mathbf{G L}_{N} \times \mathbf{G L}_{N^{\prime}}\right) / /\left(\mathbf{O}_{N} \times \mathbf{S p}_{N^{\prime}}\right)$ (say by [MFK94, Proposition 0.2]). The lemma then follows from 


\section{ORTHOGONAL AND SYMPLECTIC BUNDLES ON CURVES}

the commutative diagram below.

$$
\begin{array}{cc}
\left(\mathrm{M}_{N} \times \mathrm{M}_{N^{\prime}}\right) / /\left(\mathbf{O}_{N} \times \mathbf{S p}_{N^{\prime}}\right) \longrightarrow & \\
\cup & \Psi_{N, N^{\prime}} \\
\left(\mathbf{G L}_{N} \times \mathbf{G L}_{N^{\prime}}\right) / /\left(\mathbf{O}_{N} \times \mathbf{S p}_{N^{\prime}}\right) \stackrel{\sim}{\pi} \longrightarrow & \cup \\
\hline
\end{array}
$$

The restriction to $\left(\mathbf{G L}_{N} \times \mathbf{G L}_{N^{\prime}}\right) / /\left(\mathbf{O}_{N} \times \mathbf{S p}_{N^{\prime}}\right)$ of a map $f$ defined on the good quotient $\left(\mathrm{M}_{N} \times\right.$ $\left.\mathrm{M}_{N^{\prime}}\right) / /\left(\mathbf{O}_{N} \times \mathbf{S p}_{N^{\prime}}\right)$ must indeed be induced by a function of the form $A \in \mathbf{G L}_{N} \times \mathbf{G L}_{N^{\prime}} \mapsto$ $F\left({ }^{\mathrm{t}} A M A\right) / H\left({ }^{\mathrm{t}} A M A\right)$ with $F$ and $H$ two coprime polynomials (defined on $\Psi_{N, N^{\prime}}$ ). The equality $F\left({ }^{\mathrm{t}} A M A\right)=f(A) H\left({ }^{\mathrm{t}} A M A\right)$ finally ensures that $H$ is invertible.

2.1.3 We are now in a position to establish, again after [ABP73], the first main theorem of invariant theory for $\mathbf{O}_{N} \times \mathbf{S p}_{N^{\prime}}$. Let $V$ be a vector space of dimension $N+N^{\prime}$ endowed with the non-degenerate bilinear form $\langle\cdot, \cdot\rangle$ given by the matrix $M$. So

$$
V=V_{1} \stackrel{\perp}{\oplus} V_{2},
$$

$V_{1}$ being a quadratic space of dimension $N$ and $V_{2}$ a symplectic space of dimension $N^{\prime}$.

Theorem 2.1.4. Any linear $\mathbf{O}_{N} \times \mathbf{S p}_{N^{\prime}}$-invariant morphism $V^{\otimes 2 i} \rightarrow k$ is a linear combination of products of elementary contractions $v_{1} \otimes \cdots \otimes v_{2 i} \longmapsto\left\langle v_{l}, v_{l^{\prime}}\right\rangle$.

Let $\varphi: V^{\otimes 2 i} \rightarrow k$ be any linear $\mathbf{O}_{N} \times \mathbf{S p}_{N^{\prime}}$-invariant map, and consider the following polynomial function:

$$
f:(A, \omega) \in\left(\operatorname{End} V_{1} \oplus \operatorname{End} V_{2}\right) \times V^{\otimes 2 i} \longmapsto \varphi(A \omega) \in k .
$$

By Lemma 2.1.2, there exists a polynomial $F$ on $\left(\mathbf{S}^{2} V_{1}^{*} \oplus \boldsymbol{\Lambda}^{2} V_{2}^{*}\right) \times V^{\otimes 2 i}$, linear in the second variable, such that $f(A, \omega)=F\left({ }^{\mathrm{t}} A M A, \omega\right)$. This polynomial certainly is invariant for the natural action of $\mathbf{G L}\left(V_{1}\right) \times \mathbf{G L}\left(V_{2}\right)$ on $\left(\mathbf{S}^{2} V_{1}^{*} \oplus \mathbf{\Lambda}^{2} V_{2}^{*}\right) \times V^{\otimes 2 i}$ : for any $\Gamma \in \mathbf{G L}\left(V_{1}\right) \times \mathbf{G} \mathbf{L}\left(V_{2}\right)$, we have $F\left({ }^{\mathrm{t}} \Gamma^{-1}{ }^{\mathrm{t}} A M A \Gamma^{-1}, \Gamma \omega\right)=F\left({ }^{\mathrm{t}} A M A, \omega\right)$.

The assertion results, by polarization, from the description of linear forms on $V_{1}^{* \otimes a_{1}} \otimes V_{1}^{\otimes b_{1}} \otimes$ $V_{2}^{* \otimes a_{2}} \otimes V_{2}^{\otimes b_{2}}$ which are invariant for the action of $\mathbf{G L}\left(V_{1}\right) \times \mathbf{G L}\left(V_{2}\right): F$ is a homogeneous function of degree $i$ in its first variable, which arises from complete contractions on $\left(\mathbf{S}^{2} V_{1}^{*}\right)^{\otimes k} \otimes V_{1}^{\otimes 2 k} \otimes$ $\left(\boldsymbol{\Lambda}^{2} V_{2}^{*}\right)^{\otimes i-k} \otimes V_{2}^{\otimes 2 i-2 k}$ (via the projections $\left(\mathbf{S}^{2} V_{1}^{*} \oplus \boldsymbol{\Lambda}^{2} V_{2}^{*}\right)^{\otimes i} \times V^{\otimes 2 i} \rightarrow\left(\mathbf{S}^{2} V_{1}^{*}\right)^{\otimes k} \otimes V_{1}^{\otimes 2 k} \otimes$ $\left.\left(\Lambda^{2} V_{2}^{*}\right)^{\otimes i-k} \otimes V_{2}^{\otimes 2 i-2 k}\right)$. Since $\varphi(\omega)=F(M, \omega)$, we just have to evaluate $F$ on $M$ to conclude.

2.1.5 One easily deduces from the foregoing a family of generators for the algebra of polynomial invariants under the diagonal action (by conjugation) of $\mathbf{O}_{N} \times \mathbf{S p}_{N^{\prime}}$ on $\mathbf{M}_{N+N^{\prime}}(k)^{m}$ : according to [Pro76, $\S 7]$, it is enough to work out the behaviour of the composition, the trace and the adjunction (denoted by $A \mapsto A^{*}=M^{-1 \mathrm{t}} A M$ ) via the identification End $V \simeq V \otimes V$ induced by the bilinear pairing. If $v=v_{1}+v_{2} \in V=V_{1} \oplus V_{2}$ (cf. Paragraph 2.1.3), we have the following identities:

(i) $(v \otimes w) \circ(u \otimes t)=\langle v, t\rangle u \otimes w$;

(ii) $\operatorname{tr}(v \otimes w)=\langle v, w\rangle$;

(iii) $\left(\left(v_{1}+v_{2}\right) \otimes w\right)^{*}=w \otimes\left(v_{1}-v_{2}\right)$.

These relations allow us to translate the functions occuring in Theorem 2.1.4 in a way leading to the following statement.

Theorem 2.1.6. Any $\mathbf{O}_{N} \times \mathbf{S p}_{N^{\prime}}$-invariant function defined on $\mathrm{M}_{N+N^{\prime}}(k)^{m}$ is a polynomial in the

$$
\left(A_{1}, \ldots, A_{m}\right) \mapsto \operatorname{tr}\left(U_{j_{1}} M^{2 \delta_{1}} U_{j_{2}} M^{2 \delta_{2}} \cdots U_{j_{l}} M^{2 \delta_{l}}\right),
$$

with $l \in \mathbb{N}^{*}, U_{j_{l^{\prime}}} \in\left\{A_{j_{l^{\prime}}}, A_{j_{l^{\prime}}}^{*}\right\}$ and $\delta_{l^{\prime}} \in\{0,1\}$. 


\section{O. SERMAN}

The ring of $\mathbf{O}_{N} \times \mathbf{S p}_{N^{\prime}}$-equivariant morphisms from $\mathrm{M}_{N+N^{\prime}}(k)^{m}$ to $\mathrm{M}_{N+N^{\prime}}(k)$ is generated, as

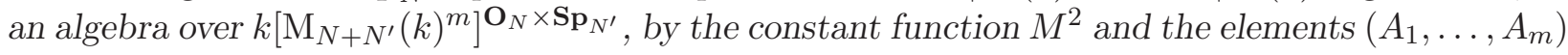
$\mapsto A_{j}$ and $\left(A_{1}, \ldots, A_{m}\right) \mapsto A_{j}^{*}$.

(The second assertion is implied by the first exactly as in [Pro76].)

\subsection{Adaptation of the main result of [Pro87]}

The main result of [Pro87] asserts that, if $R$ is a $k$-algebra with trace satisfying the $n$th CayleyHamilton identities, then there exists a natural map $R \rightarrow \mathrm{M}_{n}(A)$ which induces an isomorphism $R \rightarrow \mathrm{M}_{n}(A)^{\mathbf{G L}_{n}}$. In this section we state a similar result dealing with algebras with a trace and an antimorphism of order dividing 4 (from now on, we will write 'of order 4' instead of 'of order dividing 4').

2.2.1 Recall from [Pro87] that a $k$-algebra with trace is an algebra $R$ with a linear map tr: $R \rightarrow$ $R$ satisfying the identities $\operatorname{tr}(a) b=b \operatorname{tr}(a), \operatorname{tr}(a b)=\operatorname{tr}(b a)$ and $\operatorname{tr}(\operatorname{tr}(a) b)=\operatorname{tr}(a) \operatorname{tr}(b)$ for all $a, b \in R$. A $k$-algebra with trace and antimorphism of order 4 is an algebra with trace endowed with an antimorphism $\tau: R \rightarrow R$ of order 4 . The algebra $\mathrm{M}_{N+N^{\prime}}(B)$ of all matrices with coefficients in a commutative ring $B$ will be equipped with its natural trace together with the adjunction map (for the considered bilinear form) $\iota: A \in \mathrm{M}_{N+N^{\prime}}(k) \mapsto M^{-1}{ }^{\mathrm{t}} A M$. As soon as $N$ or $N^{\prime}$ is zero, $\iota$ is in fact of order 2 , and we could thus restrict ourselves to algebras with anti-involution.

If $R$ is such an algebra, there exists a universal morphism $j: R \rightarrow \mathrm{M}_{N+N^{\prime}}(A)$ corresponding to the functor of trace preserving representations of $R$,

$$
X_{R, N+N^{\prime}}: B \longmapsto\left\{f \in \operatorname{Hom}_{k}\left(R, \mathrm{M}_{N+N^{\prime}}(B)\right) \mid f \circ \operatorname{tr}=\operatorname{tr} \circ f\right\}
$$

(cf. [DCPRR05, $\S 2.2]$ ). The existence of this morphism easily leads to the representability of the functor $\widetilde{X}_{R, N+N^{\prime}}$ which associates to any commutative algebra $B$ the set of all trace preserving morphisms of $k$-algebras from $R$ to $\mathrm{M}_{N+N^{\prime}}(B)$ commuting with the antimorphisms. This functor is actually represented by a closed subscheme of $X_{R, N+N^{\prime}}=\operatorname{Spec}(A)$, still called $\widetilde{X}_{R, N+N^{\prime}}$ : the map $r \in R \mapsto \iota j \tau^{3}(r) \in \mathrm{M}_{N+N^{\prime}}(A)$ comes from a morphism $t: A \rightarrow A$ of order 4 , and the induced map $\tilde{\jmath}: R \rightarrow \mathrm{M}_{N+N^{\prime}}(\widetilde{A})$ (where $\widetilde{A}$ is the quotient of $A$ by the action of $t$ ) is universal among the (trace preserving) morphisms $R \rightarrow \mathrm{M}_{N+N^{\prime}}(B)$ commuting with $\tau$ and $\iota$.

The group $\mathbf{O}_{N} \times \mathbf{S p}_{N^{\prime}}$ acts by conjugation on $\mathrm{M}_{N+N^{\prime}}(B)$, inducing a right action on $\widetilde{A}$, hence an action of $\mathbf{O}_{N} \times \mathbf{S p}_{N^{\prime}}$ on $\mathbf{M}_{N+N^{\prime}}(\widetilde{A})$. The universal map $\tilde{j}$ maps $R$ to the algebra $\mathrm{M}_{N+N^{\prime}}(\widetilde{A}) \mathbf{O}_{N} \times \mathbf{S p}_{N^{\prime}}$ of $\mathbf{O}_{N} \times \mathbf{S p}_{N^{\prime}}$ equivariant morphisms from $\widetilde{X}_{R, N+N^{\prime}}$ to $\mathrm{M}_{N+N^{\prime}}(k)($ cf. [Pro87, $\left.\S 1.2]\right)$.

2.2.2 Our purpose is to adapt the main theorem of [Pro87] to this situation. To do this, we have to introduce the free product with trace $R *\langle y\rangle$ obtained from $R$ by adding a variable $y$. The next result follows from Theorem 2.1.6 as an immediate adaptation of (the first part of) Procesi's proof.

Proposition 2.2.3. If $R$ is a $k$-algebra with trace and antimorphism of order 4 , then the morphism $R * \operatorname{tr}\langle y\rangle \rightarrow \mathrm{M}_{N+N^{\prime}}(\widetilde{A})^{\mathbf{O}_{N} \times \mathbf{S p}_{N^{\prime}}}$ defined by the universal map $\tilde{j}$ and $y \mapsto M^{2}$ is onto.

Remark 2.2.4. Procesi's result actually gives an elegant condition on $R$ for the universal map $R \rightarrow$ $\mathrm{M}_{n}(A)^{\mathbf{G L}_{n}}$ to be an isomorphism. In our situation, it seems difficult to find such a nice statement. However, we can formulate the corresponding assertion as follows: the universal morphism $\tilde{j}: R \rightarrow$

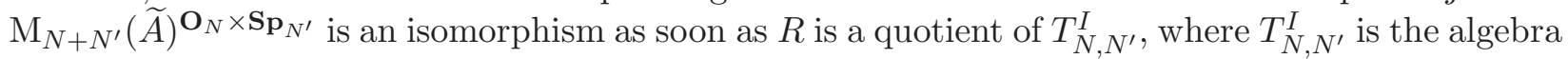
of $\mathbf{O}_{N} \times \mathbf{S p}_{N^{\prime}}$-equivariant morphisms $\mathrm{M}_{N+N^{\prime}}(k)^{I} \rightarrow \mathrm{M}_{N+N^{\prime}}(k)$ (which is therefore the maximal quotient of the free algebra (on $I \cup\{y\}$ ) with trace and antimorphism of order 4 satisfying the considered property). 


\section{ORTHOGONAL AND SYMPLECTIC BUNDLES ON CURVES}

\subsection{Generators for $k[R(Q, \alpha)]^{\Gamma_{\alpha}}$}

2.3.1 Let us go back to the quiver $Q$ and the action of $\Gamma_{\alpha}$ on its representation space $R(Q, \alpha)$. Consider the quiver $\widetilde{Q}$ obtained from $Q$ by adding one new arrow $a^{*}: \sigma\left(v^{\prime}\right) \rightarrow \sigma(v)$ for any arrow $a: v \rightarrow v^{\prime}$, where we called $\sigma$ the involution of the set of vertices fixing the $s_{i}$ and $t_{j}$, and permuting $u_{k}$ and $u_{k}^{*}$. Let $R$ (respectively $\widetilde{R}$ ) be the algebra obtained from the path algebra of the opposite quiver $Q^{\text {op }}$ (respectively $\widetilde{Q}^{\text {op }}$ ) by adding traces. There is quite a natural way to endow $\widetilde{R}$ with an antimorphism $\tau$ of order 4 whose action on the idempotents (which are associated to the constant paths) comes from the one of $\sigma: \tau$ fixes the $e_{s_{i}}$ and $e_{t_{j}}$, permutes $e_{u_{k}}$ and $e_{u_{k}^{*}}$, while it sends an arrow $a$ to $\varepsilon a^{*}$, where $\varepsilon$ equals -1 if $a$ starts from $s_{i}, u_{k}$ or $u_{k}^{*}$ and ends at $t_{j}$, and 1 otherwise.

We need here to adapt the map $\iota$ defined in Paragraph 2.2.1: $\iota$ still associates to a map its adjoint, but the bilinear pairing has to be replaced by the one represented by the matrix

$$
\Phi=\left(\begin{array}{cccc}
I_{N_{1}} & 0 & & 0 \\
0 & J_{N_{2}} & & 0 \\
0 & 0 & 0 & I_{N_{3}} \\
& & I_{N_{3}} & 0
\end{array}\right),
$$

with $N_{1}=\sum_{i=1}^{n_{1}} \alpha_{s_{i}}, N_{2}=\sum_{j=1}^{n_{2}} \alpha_{t_{j}}$ and $N_{3}=\sum_{k=1}^{n_{3}} \alpha_{u_{k}}$. Put $N=N_{1}+N_{2}+N_{3}$, and consider the decomposition of $k^{N}$ into pairwise orthogonal subspaces $k^{N_{1}} \oplus k^{N_{2}} \oplus k^{2 N_{3}}$ given by $\Phi$. Note that representations of $\widetilde{R}$ of dimension $\alpha$ commuting with $\tau$ and $\iota$ adapted to the previous decomposition correspond bijectively to representations of $R$ of the same dimension adapted to this decomposition. This allows us to identify $R(Q, \alpha)$ with the subspace of $R(\widetilde{Q}, \alpha)$ consisting of all representations which preserve the preceding antimorphisms, that is to a subspace of $\widetilde{X}_{\widetilde{R}, N}(k)$, where $\widetilde{X}_{\cdot, N}$ is the functor introduced in Paragraph 2.2.1 (once the bilinear form has been replaced by $\Phi$ ).

2.3.2 Consider now the algebra $\widetilde{S}_{n}$ defined as the quotient of $k\left[e_{s_{i}}, e_{t_{j}}, e_{u_{k}}, e_{u_{k}^{*}}\right]$ by the ideal generated by the relations $e_{v}^{2}=e_{v}, e_{v} e_{v^{\prime}}=0$ (for $v \neq v^{\prime}$ ), and $\sum_{v} e_{v}=1$. This algebra is contained in $\widetilde{R}$. The restriction of $\tau$ to this algebra is exactly the anti-involution described in Paragraph 2.3.1, and we have a fairly nice description of $\widetilde{X}_{\widetilde{S}_{n}, N}$ :

$$
\widetilde{X}_{\widetilde{S}_{n}, N}=\bigcup_{\sigma, \omega} \widetilde{X}_{\sigma, \omega}
$$

Here $\sigma$ and $\omega$ range over pairs of admissible vectors in $\mathbb{N}^{n}$ such that $\sum \sigma_{j}=N_{1}+2 N_{3}$ and $\sum \omega_{j}=N_{2}$, the component $\widetilde{X}_{\sigma, \omega}$ being isomorphic to

$$
\left(\mathbf{O}_{N_{1}+2 N_{3}} \times \mathbf{S p}_{N_{2}}\right) /\left(\prod\left(\mathbf{O}_{\sigma_{s_{i}}} \times \mathbf{S p}_{\omega_{s_{i}}}\right) \times \prod\left(\mathbf{O}_{\sigma_{t_{j}}} \times \mathbf{S p}_{\omega_{t_{j}}}\right) \times \prod\left(\mathbf{G L}_{\sigma_{u_{k}}} \times \mathbf{G L}_{\omega_{u_{k}}}\right)\right) .
$$

It induces a decomposition of $\widetilde{X}_{\widetilde{R}, N}$ as the union $\bigcup_{\sigma, \omega} \varpi^{-1} \widetilde{X}_{\sigma, \omega}$, where we have called $\varpi: \widetilde{X}_{\widetilde{R}, N} \rightarrow$ $\widetilde{X}_{\widetilde{S}_{n}, N}$ the map induced by the inclusion $\widetilde{S}_{n} \subset \widetilde{R}$. By applying the argument of [LBP90, $\left.\S 3\right]$ to the component corresponding to the dimension vectors $\sigma$ and $\omega$ whose coordinates are $\sigma_{s_{i}}=\alpha_{s_{i}}, \sigma_{t_{j}}=0$, $\sigma_{u_{k}}=\alpha_{u_{k}}, \omega_{t_{j}}=\alpha_{t_{j}}$ and $\omega_{s_{i}}=\omega_{u_{k}}=0$, we get the following expected assertion.

THEOREM 2.3.3. The algebra of polynomials on $R(Q, \alpha)$ invariant under the action of $\prod \mathbf{O}_{\alpha_{s_{i}}} \times$ $\prod \mathbf{S p}_{\alpha_{t_{j}}} \times \prod \mathbf{G L}_{\alpha_{u_{k}}}$ is generated by the functions

$$
\left(f_{a}\right)_{a} \longmapsto \operatorname{tr}\left(f_{\tilde{a}_{p}} \cdots f_{\tilde{a}_{1}}\right),
$$

where $\tilde{a}_{i}$ is an arrow in the associated quiver $\widetilde{Q}$ equal to either $a_{i}$ or $a_{i}^{*}$ in such a way that $\left(\tilde{a}_{1}, \ldots, \tilde{a}_{p}\right)$ 


\section{O. Serman}

forms an oriented path in that quiver, and $f_{\tilde{a}_{i}}$ means $f_{a_{i}}$ or its adjoint according to whether $\tilde{a}_{i}$ is $a_{i}$ or $a_{i}^{*}$.

Remark 2.3.4. It is now easy to deal with the case where we let the whole linear group act (by conjugation) above some of the unpaired vertices: let us call $r_{1}, \ldots, r_{n_{4}}$ these vertices, $Q^{\prime}$ the quiver obtained by adding $n_{4}$ new vertices $r_{l}^{*}$ and $\alpha^{\prime} \in \mathbb{N}^{n_{1}+n_{2}+2\left(n_{3}+n_{4}\right)}$ the admissible vector naturally deduced from any given admissible dimension vector $\alpha \in \mathbb{N}^{n_{1}+n_{2}+2 n_{3}+n_{4}}$. The group

$$
\Gamma_{\alpha}=\prod \mathbf{O}_{\alpha_{s_{i}}} \times \prod \mathbf{S p}_{\alpha_{t_{j}}} \times \prod \mathbf{G L}_{\alpha_{u_{k}}} \times \prod \mathbf{G L}_{\alpha_{r_{l}}}
$$

acts on $R(Q, \alpha)$ and $R\left(Q^{\prime}, \alpha^{\prime}\right)$ (the action of $g \in \mathbf{G L}_{\alpha_{r_{l}}}$ on $r_{l}^{*}$ being $f \mapsto{ }^{\mathrm{t}} g^{-1} f^{\mathrm{t}} g$ ), and the $\Gamma_{\alpha^{-}}$ equivariant projection $k\left[R\left(Q^{\prime}, \alpha^{\prime}\right)\right] \rightarrow k[R(Q, \alpha)]$ allows us to compute the ring of $\Gamma_{\alpha}$-invariants of $k[R(Q, \alpha)]$.

\section{Local study of the forgetful map}

In order to simplify the local study of $\mathcal{M}_{\mathbf{S O}_{r}} \longrightarrow \mathcal{M}_{\mathbf{S L}_{r}}$ it is convenient to investigate separately the injective morphism $\mathcal{M}_{\mathbf{O}_{r}} \longrightarrow \mathcal{M}_{\mathbf{G L}_{r}}$ and the natural map from $\mathcal{M}_{\mathbf{S O}_{r}}$ to the irreducible component $\mathcal{M}_{\mathbf{O}_{r}}^{\mathcal{O}}$ of $\mathcal{M}_{\mathbf{O}_{r}}$ consisting of all orthogonal bundles with trivial determinant. This distinction seems to be quite valuable since the direct differential study of $\mathcal{M}_{\mathbf{S O}_{r}}$ would involve invariant theory for special orthogonal groups, which is far more difficult to deal with (see $\S 3.2$ ). We show here that the former is an embedding, while the latter is an isomorphism (respectively a 2-sheeted cover) when $r$ is odd (respectively even).

\subsection{Differential behaviour of $\mathcal{M}_{\mathrm{O}_{r}} \longrightarrow \mathcal{M}_{\mathrm{GL}_{r}}$}

3.1.1 Let us now briefly point out the classical way to analyse the local behaviour of $\mathcal{M}_{\mathbf{O}_{r}} \longrightarrow$ $\mathcal{M}_{\mathbf{G L}_{r}}$. Recall first that this application arises as a quotient (by a general linear group $\Gamma=\mathbf{G L}_{M}$ ) of an equivariant map between two well-known parameter schemes $R_{\mathbf{O}_{r}} \longrightarrow R_{\mathbf{G L}_{r}}$ (cf. [BLS98, Lemma 7.3]). Luna's étale slice theorem and deformation theory then allow us to grasp the local structure of these good quotients (cf. [KLS06, § 2.5]): at any polystable vector bundle $E, \mathcal{M}_{\mathbf{G L}_{r}}$ is étale locally isomorphic to an étale neighbourhood of the origin in the good quotient

$$
\operatorname{Ext}^{1}(E, E) / / \operatorname{Aut}_{\mathbf{G L}}(E)
$$

while $\mathcal{M}_{\mathbf{O}_{r}}$ is, at any unitary orthogonal bundle $P$, étale locally isomorphic to an étale neighbourhood of the origin in

$$
H^{1}(C, \operatorname{Ad}(P)) / / \operatorname{Aut}_{\mathbf{O}_{r}}(P),
$$

where $\operatorname{Ad}(P)$ stands for the vector bundle $P \times \mathbf{O}_{r} \mathfrak{s o}_{r}$ associated to the adjoint representation of $\mathbf{O}_{r}$, which is nothing other than the vector bundle of germs of endomorphisms $f$ of $E$ such that $\sigma f+f^{*} \sigma=0$, where $\sigma: E \rightarrow E^{*}$ is the symmetric isomorphism given by the quadratic structure on $E$; in other words, the adjoint vector bundle $\operatorname{Ad}(P)$ is canonically isomorphic to $\Lambda^{2} E^{*}$.

Then, if $P \in \mathcal{M}_{\mathbf{O}_{r}}$ is a unitary orthogonal bundle with associated vector bundle $E \in \mathcal{M}_{\mathbf{G L}_{r}}$, the application $\mathcal{M}_{\mathbf{O}_{r}} \longrightarrow \mathcal{M}_{\mathbf{G L}_{r}}$ coincides at $P$, through the preceding local isomorphisms, with the natural map

$$
H^{1}(C, \operatorname{Ad}(P)) / / \operatorname{Aut}_{\mathbf{O}_{r}}(P) \longrightarrow \operatorname{Ext}^{1}(E, E) / / \operatorname{Aut}_{\mathbf{G} \mathbf{L}_{r}}(E)
$$

at the origin. In particular, the corresponding tangent maps are identified.

3.1.2 A more explicit description of the vector spaces $H^{1}(C, \operatorname{Ad}(P))$ and $\operatorname{Ext}^{1}(E, E)$ is then strongly needed in order to understand their quotients; this will allow us to show that the quotient 


\section{ORTHOGONAL AND SYMPLECTIC BUNDLES ON CURVES}

$H^{1}(C, \operatorname{Ad}(P)) / / \operatorname{Aut}_{\mathbf{O}_{r}}(P)$ is in fact a closed subscheme of $\operatorname{Ext}^{1}(E, E) / / \operatorname{Aut}_{\mathbf{G L}_{r}}(E)$, which implies our main theorem.

According to Remark 1.3 (i), the orthogonal structure on the polystable vector bundle $E$ associated to any closed point $P \in \mathcal{M}_{\mathbf{O}_{r}}$ gives rise to a splitting of $E$ as a direct orthogonal sum of the form

$$
E=\bigoplus_{i=1}^{n_{1}} E_{i}^{(1)} \oplus \bigoplus_{j=1}^{n_{2}} E_{j}^{(2)} \oplus \bigoplus_{k=1}^{n_{3}} E_{k}^{(3)}
$$

where each direct summand $E_{l}^{(a)}$ (respectively isomorphic to $F_{i}^{(1)} \otimes V_{i}^{(1)}, F_{j}^{(2)} \otimes V_{j}^{(2)}$ or $\left(F_{k}^{(3)} \oplus\right.$ $\left.F_{k}^{(3)_{*}}\right) \otimes V_{k}^{(3)}$; see Equation (1.2.1)) is an orthogonal bundle via an induced symmetric isomorphism denoted by $\sigma_{l}^{(a)}: E_{l}^{(a)} \rightarrow E_{l}^{(a)_{*}}$. The two isotropy groups $\operatorname{Aut}_{\mathbf{G L}_{r}}(E)$ and $\operatorname{Aut}_{\mathbf{O}_{r}}(P)$ have been identified in $\S 1$.

The space $\operatorname{Ext}^{1}(E, E)$ splits into a direct sum of the spaces $\operatorname{Ext}^{1}\left(E_{i}^{(k)}, E_{j}^{(l)}\right)$, and each of these summands is isomorphic to $\operatorname{Ext}^{1}\left(F_{i}^{(k)}, F_{j}^{(l)}\right) \otimes \operatorname{Hom}\left(V_{i}^{(k)}, V_{j}^{(l)}\right)$ when neither $k$ nor $l$ equals 3 , or to a sum of summands of this form otherwise. The isotropy groups act on each of those spaces via the natural actions of $\mathbf{G} \mathbf{L}(V) \times \mathbf{G} \mathbf{L}\left(V^{\prime}\right)$ on $\operatorname{Hom}\left(V, V^{\prime}\right)$.

An element

$$
\omega=\sum \omega_{i, j}^{(k, l)} \in \operatorname{Ext}^{1}(E, E) \simeq \bigoplus \operatorname{Ext}^{1}\left(E_{i}^{(k)}, E_{j}^{(l)}\right)
$$

belongs to the space $H^{1}(C, \operatorname{Ad}(P))$ if and only if $\omega_{i, i}^{(k, k)} \in H^{1}\left(C, \Lambda^{2} E_{i}^{(k)_{*}}\right) \subset \operatorname{Ext}^{1}\left(E_{i}^{(k)}, E_{i}^{(k)}\right)$ and, for $(i, k) \neq(j, l), \sigma_{j}^{(l)} \omega_{i, j}^{(k, l)}+\omega_{j, i}^{(l, k) *} \sigma_{i}^{(k)}=0$. So, identifying $\operatorname{Ext}^{1}\left(E_{i}^{(k)}, E_{j}^{(l)}\right)$ with its image in $\operatorname{Ext}^{1}\left(E_{i}^{(k)}, E_{j}^{(l)}\right) \oplus \operatorname{Ext}^{1}\left(E_{j}^{(l)}, E_{i}^{(k)}\right)$ by the application $\omega_{i, j}^{(k, l)} \mapsto \omega_{i, j}^{(k, l)}-\sigma_{i}^{(k)}-1 \omega_{i, j}^{(k, l)} * \sigma_{j}^{(l)}$, it appears that $H^{1}(C, \operatorname{Ad}(P))$ is the subspace of $\operatorname{Ext}^{1}(E, E)$ isomorphic to the direct sum

$$
\bigoplus_{k}\left(\bigoplus_{i} H^{1}\left(C, \Lambda^{2} E_{i}^{(k) *}\right) \oplus \bigoplus_{i<j} \operatorname{Ext}^{1}\left(E_{i}^{(k)}, E_{j}^{(k)}\right)\right) \oplus \bigoplus_{k<l} \bigoplus_{i, j} \operatorname{Ext}^{1}\left(E_{i}^{(k)}, E_{j}^{(l)}\right) .
$$

Each one of the diagonal summands is more precisely expressed as

$$
\begin{aligned}
& H^{1}\left(C, \boldsymbol{\Lambda}^{2} E_{i}^{(1)_{*}}\right)=\left(H^{1}\left(C, \mathbf{S}^{2} F_{i}^{(1) *}\right) \otimes \mathfrak{s o}\left(V_{i}^{(1)}\right)\right) \oplus\left(H^{1}\left(C, \boldsymbol{\Lambda}^{2} F_{i}^{(1) *}\right) \otimes \mathbf{S}^{2} V_{i}^{(1)_{*}}\right), \\
& H^{1}\left(C, \boldsymbol{\Lambda}^{2} E_{j}^{(2) *}\right)=\left(H^{1}\left(C, \Lambda^{2} F_{j}^{(2) *}\right) \otimes \mathfrak{s p}\left(V_{j}^{(2)}\right)\right) \oplus\left(H^{1}\left(C, \mathbf{S}^{2} F_{j}^{(2) *}\right) \otimes \Lambda^{2} V_{j}^{(2) *}\right), \\
& H^{1}\left(C, \boldsymbol{\Lambda}^{2} E_{k}^{(3) *}\right)=\left(\operatorname{Ext}^{1}\left(F_{k}^{(3)}, F_{k}^{(3)}\right) \otimes \mathfrak{g l}\left(V_{k}^{(3)}\right)\right) \oplus\left(H^{1}\left(C, \mathbf{S}^{2} F_{k}^{(3) *}\right) \otimes \boldsymbol{\Lambda}^{2} V_{k}^{(3) *}\right) \\
& \oplus\left(H^{1}\left(C, \boldsymbol{\Lambda}^{2} F_{k}^{(3) *}\right) \otimes \mathbf{S}^{2} V_{k}^{(3) *}\right) \oplus\left(H^{1}\left(C, \mathbf{S}^{2} F_{k}^{(3)}\right) \otimes \boldsymbol{\Lambda}^{2} V_{k}^{(3) *}\right) \\
& \oplus\left(H^{1}\left(C, \Lambda^{2} F_{k}^{(3)}\right) \otimes \mathbf{S}^{2} V_{k}^{(3) *}\right) .
\end{aligned}
$$

Here we have identified the space $\operatorname{Ext}^{1}\left(F_{k}^{(3)}, F_{k}^{(3)}\right) \otimes \mathfrak{g l}\left(V_{k}^{(3)}\right)$ with its image in $\left(\operatorname{Ext}^{1}\left(F_{k}^{(3)}, F_{k}^{(3)}\right) \oplus\right.$ $\left.\operatorname{Ext}^{1}\left(F_{k}^{(3)_{*}}, F_{k}^{(3) *}\right)\right) \otimes \mathfrak{g l}\left(V_{k}^{(3)}\right)$ by the map $\omega \otimes \varphi \mapsto \omega \otimes \varphi-\omega^{*} \otimes{ }^{\mathrm{t}} \varphi$. Note that the dimensions of all the extension spaces under consideration are trivially available.

3.1.3 This rather intricate situation suitably expresses itself in terms of representations of quivers. Indeed, let us consider the quiver $\mathcal{Q}$ whose set of vertices is

$$
\mathcal{Q}_{0}=\left\{s_{1}^{(1)}, \ldots, s_{n_{1}}^{(1)}, s_{1}^{(2)}, \ldots, s_{n_{2}}^{(2)}, s_{1}^{(3)}, s_{1}^{\left(3^{*}\right)}, \ldots, s_{n_{3}}^{(3)}, s_{n_{3}}^{\left(3^{*}\right)}\right\},
$$

these vertices being connected by $\operatorname{dim} \operatorname{Ext}^{1}\left(F_{i}^{(k)}, F_{j}^{(l)}\right)$ arrows from $s_{i}^{(k)}$ to $s_{j}^{(l)}$ (where we have set $F_{i}^{\left(3^{*}\right)}=F_{i}^{\left.(3)_{*}\right)}$. Next define $\alpha \in \mathbb{N}^{n_{1}+n_{2}+2 n_{3}}$ according to the dimensions of the corresponding vector spaces $V_{l}^{(a)}$. The $\operatorname{Aut}_{\mathbf{G L}}(E)$-module $\operatorname{Ext}^{1}(E, E)$ is then exactly the $\mathbf{G L}(\alpha)$-module $R(\mathcal{Q}, \alpha)$ 


\section{O. Serman}

composed of all the representations of $\mathcal{Q}$ of dimension $\alpha$, and the result of [LBP90] recalled earlier provides us with a description of the algebra $k\left[\operatorname{Ext}^{1}(E, E)\right]^{\operatorname{Aut}_{\mathbf{G L}_{r}}(E)}$.

Since the inclusion $H^{1}(C, \operatorname{Ad}(P)) \hookrightarrow \operatorname{Ext}^{1}(E, E)$ is an $\operatorname{Aut}_{\mathbf{O}_{r}}(P)$-equivariant application, we have an exact sequence

$$
k\left[\operatorname{Ext}^{1}(E, E)\right]^{\operatorname{Aut}_{\mathbf{O}_{r}}(P)} \rightarrow k\left[H^{1}(C, \operatorname{Ad}(P))\right]^{\operatorname{Aut}_{\mathbf{O}_{r}}(P)} \rightarrow 0 .
$$

This sequence and Theorem 2.3.3 immediately result in a set of generators for the invariant algebra $k\left[H^{1}(C, \operatorname{Ad}(P))\right]^{\operatorname{Aut}_{\mathbf{O}_{r}}(P)}$, namely the $\left(f_{a}\right)_{a} \mapsto \operatorname{tr}\left(f_{\tilde{a}_{p}} \cdots f_{\tilde{a}_{1}}\right)$, where $f_{\tilde{a}_{i}}$ stands for either $f_{a_{i}}$ or its adjoint map. Now, according to the direct sum in (3.1.2.2), $H^{1}(C, \operatorname{Ad}(P))$ is a subspace of $R(\mathcal{Q}, \alpha)$ made up of representations having the following property. If $f_{a}: V_{v} \rightarrow V_{v^{\prime}}$ denotes the map associated to an arrow $a: v \rightarrow v^{\prime}$, then its adjoint map $f_{a}^{*}: V_{v^{\prime}}^{*} \rightarrow V_{v}^{*}$ is, up to the sign, the map associated to one of the arrows going from $v^{\prime}$ to $v$. So the algebra $k\left[H^{1}(C, \operatorname{Ad}(P))\right]^{\operatorname{Aut}_{\mathbf{O}_{r}(P)}}$ is generated by traces along oriented cycles in the quiver $\mathcal{Q}$. This exactly means that the application $k\left[\operatorname{Ext}^{1}(E, E)\right]^{\operatorname{Aut}_{\mathbf{G L}_{r}}(E)} \rightarrow k\left[H^{1}(C, \operatorname{Ad}(P))\right]^{\operatorname{Aut}_{\mathbf{O}_{r}}(P)}$ is onto.

In view of what has been discussed in Paragraph 3.1.1, this proves the injectivity of the tangent map of $\mathcal{M}_{\mathbf{O}_{r}} \longrightarrow \mathcal{M}_{\mathbf{G L}_{r}}$ at any $P$. Since, as we have already seen in Paragraph 1.2, the map $\mathcal{M}_{\mathbf{O}_{r}}(k) \rightarrow \mathcal{M}_{\mathbf{G L}_{r}}(k)$ is also injective, this finishes the proof of the first part of our main theorem.

Remark 3.1.4. Part (iii) of the main theorem is proved in the very same way: the point is that any closed point $P$ of $\mathcal{M}_{\mathbf{S p}_{2 r}}$ corresponds to a symplectic bundle of the form

$$
E=\bigoplus_{i}\left(F_{i}^{(1)} \otimes V_{i}^{(1)}\right) \oplus \bigoplus_{j}\left(F_{j}^{(2)} \otimes V_{j}^{(2)}\right) \oplus \bigoplus_{k}\left(\left(F_{k}^{(3)} \oplus F_{k}^{(3) *}\right) \otimes V_{k}^{(3)}\right),
$$

where $\left(F_{i}^{(1)}\right)_{i}$ (respectively $\left(F_{j}^{(2)}\right)_{j}$, respectively $\left(F_{k}^{(3)}\right)_{k}$ ) is a family of mutually non-isomorphic symplectic (respectively orthogonal, respectively not self-dual) bundles (which are stable as vector bundles), and $\left(V_{i}^{(1)}\right)_{i}$ (respectively $\left(V_{j}^{(2)}\right)_{j}$, respectively $\left(V_{k}^{(3)}\right)_{k}$ ) a family of quadratic (respectively symplectic, respectively endowed with a non-degenerate bilinear pairing) vector spaces $\left(F_{k}^{(3)} \oplus F_{k}^{(3) *}\right.$ now being equipped with the standard symplectic form). Let us denote by $\sigma: E \rightarrow E^{*}$ the resulting symplectic form on $E$. The bundle $\operatorname{Ad}(P)$ is now isomorphic to the bundle of germs of symmetric endomorphisms of $E$ (that is, endomorphisms verifying $\sigma f+f^{*} \sigma=0$ ), and both the space $H^{1}(C, \operatorname{Ad}(P))$ and the isotropy group $\operatorname{Aut}_{\mathbf{S p}_{2 r}}(P)$ can be described in a manner analogous to that of Paragraph 3.1.2 (one only has to switch the factors $\boldsymbol{\Lambda}^{2} F_{l}^{(a)}{ }_{*}$ and $\mathbf{S}^{2} F_{l}^{(a) *}$, and of course to redefine in the obvious way every map of the form $\left.\operatorname{Ext}^{1}\left(F, F^{\prime}\right) \rightarrow \operatorname{Ext}^{1}\left(F, F^{\prime}\right) \oplus \operatorname{Ext}^{1}\left(F^{*}, F^{*}\right)\right)$. Theorem 2.3.3 then allows us to conclude again.

\subsection{About $\mathcal{M}_{\mathrm{SO}_{r}} \longrightarrow \mathcal{M}_{\mathrm{O}_{r}}$}

3.2.1 We have already recalled in Remark 1.6 how to compute the fibres of the finite morphism from $\mathcal{M}_{\mathbf{S O}_{r}}$ onto $\mathcal{M}_{\mathbf{O}_{r}}^{\mathcal{O}}=\operatorname{det}^{-1}\left(\mathcal{O}_{C}\right)$. Luna's theorem reduces once again the differential study of this application to an invariant computation: the tangent map of $\mathcal{M}_{\mathbf{S O}_{r}} \longrightarrow \mathcal{M}_{\mathbf{O}_{r}}$ at $P \in \mathcal{M}_{\mathbf{S O}_{r}}$ is indeed identified with that of $H^{1}(C, \operatorname{Ad}(P)) / / \operatorname{Aut}_{\mathbf{S O}_{r}}(P) \rightarrow H^{1}(C, \operatorname{Ad}(P)) / / \operatorname{Aut}_{\mathbf{O}_{r}}(P)$ (at the origin).

This easily implies that, when $r$ is odd, the map $\mathcal{M}_{\mathbf{S O}_{r}} \longrightarrow \mathcal{M}_{\mathbf{O}_{r}}^{\mathcal{O}}$ is an isomorphism.

3.2.2 Let us consider now the even case. The morphism $\mathcal{M}_{\mathbf{S O}_{r}} \longrightarrow \mathcal{M}_{\mathbf{O}_{r}}$ is then a 2-sheeted cover, which is étale above the locus of points having two antecedents. A branched point corresponds to an orthogonal polystable bundle $E$ containing at least one subbundle isomorphic to $F_{i}^{(1)} \otimes V_{i}^{(1)}$ 


\section{ORTHOGONAL AND SYMPLECTIC BUNDLES ON CURVES}

where $F_{i}^{(1)}$ is an orthogonal bundle of odd rank: we then have to understand the inclusion

$$
k\left[H^{1}(C, \operatorname{Ad}(P))\right]^{\operatorname{Aut}_{\mathbf{O}_{r}}(P)} \hookrightarrow k\left[H^{1}(C, \operatorname{Ad}(P))\right]^{\operatorname{Aut}_{\mathbf{s O}_{r}}(P)} .
$$

It is easy to produce a primitive element for the generic extension. First note that the vector space $W$ obtained as the direct sum of the vector spaces $V_{i}^{(1)}$ corresponding to the orthogonal bundles $F_{i}^{(1)}$ of odd rank has even dimension, and has an orthogonal structure inherited from the ones of the spaces $V_{i}^{(1)}$. The space composed of all the antisymmetric endomorphisms of $W$ may be identified with a direct summand of $H^{1}(C, \operatorname{Ad}(P))$, and mapping any element $\omega \in H^{1}(C, \operatorname{Ad}(P))$ to the pfaffian of the endomorphism of $W$ induced by $\omega$ defines a function belonging to $k\left[H^{1}(C, \operatorname{Ad}(P))\right]^{\operatorname{Aut}_{\mathbf{S O}_{r}}(P)}$ which is not $\operatorname{Aut}_{\mathbf{O}_{r}}(P)$-invariant; this function certainly generates the generic extension.

It is more difficult to give a convenient description of this algebra: in the (simplest) case where $P$ is isomorphic to $\mathcal{O} \otimes V$, with $V$ a quadratic vector space of even dimension $r$, we have to understand the action of $\operatorname{Aut}_{\mathbf{S O}_{r}}(P) \simeq \mathbf{S O}_{r}$ on $H^{1}(C, \operatorname{Ad}(P)) \simeq H^{1}(C, \mathcal{O}) \otimes \mathfrak{s o}(V)$. This can be solved again thanks to Procesi's trick (cf. Paragraph 2.1.5): the computation has been carried out in [ATZ95], and provides a set of generators for the $k\left[H^{1}(C, \operatorname{Ad}(P))\right]^{\operatorname{Aut}_{\mathbf{O}_{r}}(P)}$-algebra $k\left[H^{1}(C, \operatorname{Ad}(P))\right]^{\operatorname{Aut}_{\mathbf{S O}_{r}}(P)}$ in terms of polarized pfaffians.

Let us finally mention that in the general case we can easily infer from the main result of [Lop06] a family of generators for $k\left[H^{1}(C, \operatorname{Ad}(P))\right]^{\operatorname{Aut}_{\mathbf{S O}_{r}}(P)}$, which are also obtained as polarized pfaffians.

\subsection{About the multiplicity at stable points}

3.3.1 The discussion in Paragraph 3.1.1 contains in fact a more precise statement, related to the completed local rings of $\mathcal{M}_{\mathbf{O}_{r}}$ and $\mathcal{M}_{\mathbf{G L}_{r}}$. Indeed, if $P$ defines a point of $\mathcal{M}_{\mathbf{O}_{r}}$ whose image in $\mathcal{M}_{\mathbf{G L}_{r}}$ corresponds to $E$, we have the following commutative diagram

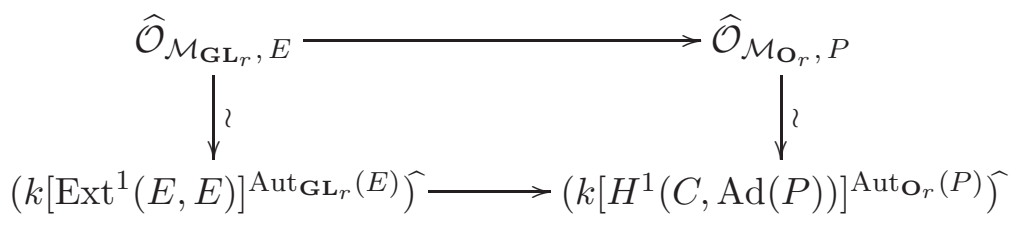

where the rings of the second row are the completions of the local rings (of the involved algebras of invariants) at the origin. This description of the completed local rings of $\mathcal{M}_{\mathbf{O}_{r}}$ provides us with additional information about the local structure of $\mathcal{M}_{\mathbf{O}_{r}}$, at least at the points where the situation is not too bad (see [Las96] for the case of $\mathcal{M}_{\mathbf{G L}_{r}}$ ): the more we know about the second main theorem of invariant theory for the isotropy group of $P$, the easier our calculations will be.

3.3.2 Let $P$ be an orthogonal bundle whose underlying vector bundle is of the form $E=E_{1} \oplus E_{2}$, with $E_{1}$ and $E_{2}$ two non-isomorphic GL-stable orthogonal bundles. The description of the inclusion $H^{1}(C, \operatorname{Ad}(P)) \hookrightarrow \operatorname{Ext}^{1}(E, E)$ given in (3.1.2.2) here reduces to the following.

$$
\begin{aligned}
& H^{1}(C, \operatorname{Ad}(P))=H^{1}\left(C, \Lambda^{2} E_{1}{ }^{*}\right) \oplus \quad \operatorname{Ext}^{1}\left(E_{1}, E_{2}\right) \quad \oplus H^{1}\left(C, \Lambda^{2} E_{2}{ }^{*}\right) \\
& \operatorname{Ext}^{1}(E, E)=\operatorname{Ext}^{1}\left(E_{1}, E_{1}\right) \quad \oplus \operatorname{Ext}^{1}\left(E_{1}, E_{2}\right) \oplus \operatorname{Ext}^{1}\left(E_{2}, E_{1}\right) \oplus \operatorname{Ext}^{1}\left(E_{2}, E_{2}\right)
\end{aligned}
$$

The isotropy subgroup $\operatorname{Aut}_{\mathbf{O}_{r}}(P)$, isomorphic to $\mathbb{Z} / 2 \mathbb{Z} \times \mathbb{Z} / 2 \mathbb{Z}$, acts trivially on $H^{1}\left(C, \Lambda^{2} E_{i}^{*}\right)$, and by multiplication by \pm 1 on $\operatorname{Ext}^{1}\left(E_{1}, E_{2}\right)$ (while $\left(\alpha_{1}, \alpha_{2}\right) \in \operatorname{Aut}_{\mathbf{G L}_{r}}(E) \simeq \mathbb{G}_{m} \times \mathbb{G}_{m}$ acts on $\operatorname{Ext}^{1}\left(E_{i}, E_{j}\right)$ by $\left.\alpha_{j} \alpha_{i}^{-1}\right)$.

Let $\left(X_{k}^{(i)}\right)_{k}$ (respectively $\left.\left(Y_{l}\right)_{l}\right)$ be a basis of $H^{1}\left(C, \Lambda^{2} E_{i}^{*}\right)^{*}\left(\operatorname{respectively~} \operatorname{Ext}^{1}\left(E_{1}, E_{2}\right)^{*} \subset\right.$ $\left.H^{1}(C, \operatorname{Ad}(P))^{*}\right)$. Then $k\left[H^{1}(C, \operatorname{Ad}(P))\right]^{\operatorname{Aut}_{\mathbf{O}_{r}}(P)}$ is the subring of $k\left[X_{k}^{(i)}, Y_{l}\right]$ generated by the $X_{k}^{(i)}$ 


\section{O. Serman}

and the products $Y_{l} Y_{l^{\prime}}$; if $\mathcal{V}$ denotes the affine cone over the Veronese variety $\mathbb{P}\left(\operatorname{Ext}^{1}\left(E_{1}, E_{2}\right)\right) \subset$ $\mathbb{P}\left(\mathbf{S}^{2} \operatorname{Ext}^{1}\left(E_{1}, E_{2}\right)\right)$, we get the following isomorphism:

$$
\operatorname{Spec}\left(k\left[H^{1}(C, \operatorname{Ad}(P))\right]^{\operatorname{Aut}_{\mathbf{O}_{r}}(P)}\right) \stackrel{\sim}{\longrightarrow}\left(H^{1}\left(C, \boldsymbol{\Lambda}^{2} E_{1}^{*}\right) \oplus H^{1}\left(C, \boldsymbol{\Lambda}^{2} E_{2}{ }^{*}\right)\right) \times \mathcal{V} .
$$

Using the identification $\widehat{\mathcal{O}}_{\mathcal{M}_{\mathbf{O}_{r}}, P} \simeq\left(k\left[H^{1}(C, \operatorname{Ad}(P))\right]^{\mathrm{Aut}_{\mathbf{O}_{r}}(P)}\right)^{\widehat{w}}$ we have the following result.

Corollary 3.3.3. The tangent space to $\mathcal{M}_{\mathbf{O}_{r}}$ at a point $P$ given as the direct sum $E_{1} \oplus E_{2}$ of two non-isomorphic stable orthogonal bundles is isomorphic to

$$
H^{1}\left(C, \Lambda^{2} E_{1}^{*}\right) \oplus H^{1}\left(C, \Lambda^{2} E_{2}^{*}\right) \oplus\left(\mathbf{S}^{2} \operatorname{Ext}^{1}\left(E_{1}, E_{2}\right)\right),
$$

and the multiplicity of $\mathcal{M}_{\mathbf{O}_{r}}$ at this point is equal to $2^{r_{1} r_{2}(g-1)-1}$, where $r_{i}$ is the rank of $E_{i}$.

Remark 3.3.4. (i) The general case of a stable point $P \in \mathcal{M}_{\mathbf{O}_{r}}$ is more difficult: such a bundle corresponds to a vector bundle which splits as the direct sum of $n$ mutually non-isomorphic $\mathbf{G L}_{r_{i}}{ }^{-}$ stable orthogonal bundles. We can use Theorem 2.3.3 to try to get some additional information about the local structure at $P$, for instance by computing the multiplicity at this point. One can easily check that, if $n=3$ (respectively 4 ), this multiplicity is equal to $2 \prod_{i<j} 2^{r_{i} r_{j}(g-1)-1}$ (respectively $\left.8 \prod_{i<j} 2^{r_{i} r_{j}(g-1)-1}\right)$.

(ii) It is not hard to deal with the case of an orthogonal bundle of the form $F \oplus F^{*}$ with $F \not F^{*}$ : we see that $\mathcal{M}_{\mathbf{O}_{r}}$ is, at such a point, étale locally isomorphic to $\operatorname{Ext}^{1}(F, F) \oplus \mathcal{S}$, where $\mathcal{S}$ is the affine cone over the Segre variety

$$
\mathbb{P}\left(H^{1}\left(C, \boldsymbol{\Lambda}^{2} F^{*}\right)\right) \times \mathbb{P}\left(H^{1}\left(C, \Lambda^{2} F\right)\right) \subset \mathbb{P}\left(H^{1}\left(C, \Lambda^{2} F^{*}\right) \otimes H^{1}\left(C, \Lambda^{2} F\right)\right) .
$$

\section{ACKNOWLEDGEMENTS}

I am very grateful to A. Beauville for having introduced me to this question, and for his helpful comments and advice during the elaboration of this work.

\section{REFERENCES}

ATZ95 H. Aslaksen, E.-C. Tan and C.-B. Zhu, Invariant theory of special orthogonal groups, Pacific J. Math. 168 (1995), 207-215.

ABP73 M. Atiyah, R. Bott and V. K. Patodi, On the heat equation and the index theorem, Invent. Math. 19 (1973), 279-330.

BS02 V. Balaji and C. S. Seshadri, Semistable principal bundles - I (characteristic zero), J. Algebra 258 (2002), 321-347.

BLS98 A. Beauville, Y. Laszlo and C. Sorger, The Picard group of the moduli of G-bundles on a curve, Compositio Math. 112 (1998), 183-216.

Bho84 U. N. Bhosle, Moduli of orthogonal and spin bundles over hyperelliptic curves, Compositio Math. 51 (1984), 15-40.

DCPRR05 C. De Concini, C. Procesi, N. Reshetikhin and M. Rosso, Hopf algebras with trace and representations, Invent. Math. 161 (2005), 1-44.

Gir71 J. Giraud, Cohomologie non abélienne, Grundlehren der mathematischen Wissenschaften, vol. 179 (Springer, Berlin, 1971).

KLS06 D. Kaledin, M. Lehn and C. Sorger, Singular symplectic moduli spaces, Invent. Math. 164 (2006), 591-614.

Las96 Y. Laszlo, Local structure of the moduli space of vector bundles over curves, Comment. Math. Helv. 71 (1996), 373-401.

LBP90 L. Le Bruyn and C. Procesi, Semisimple representations of quivers, Trans. Amer. Math. Soc. 317 (1990), 585-598. 


\section{ORTHOGONAL AND SYMPLECTIC BUNDLES ON CURVES}

Lop06 A. A. Lopatin, Invariants of quivers under the action of classical groups, Preprint (2006), arXiv:math.RT/0608750.

MFK94 D. Mumford, J. Fogarty and F. Kirwan, Geometric invariant theory, third edition, Ergebnisse der Mathematik und ihrer Grenzgebiete, vol. 34 (Springer, Berlin, 1994).

Pro76 C. Procesi, The invariant theory of $n \times n$ matrices, Adv. Math. 19 (1976), 306-381.

Pro87 C. Procesi, A formal inverse to the Cayley-Hamilton theorem, J. Algebra 107 (1987), 63-74.

Ram81 S. Ramanan, Orthogonal and spin bundles over hyperelliptic curves, Proc. Indian Acad. Sci. Math. Sci. 90 (1981), 151-166.

Ram96 A. Ramanathan, Moduli for principal bundles over algebraic curves. I and II, Proc. Indian Acad. Sci. Math. Sci. 106 (1996), 301-328, 421-449.

Olivier Serman serman@math.unice.fr

Laboratoire J.-A. Dieudonné, UMR 6621 du CNRS, Université de Nice, Parc Valrose, F-06108 Nice cedex 02, France 